\title{
Typologie des exploitations agricoles productrices du mil et niébé pluvial dans la commune rurale de Karma
}

\author{
Yacouba Ali RAZINATOU ${ }^{1 *}$, Soumana BOUBACAR ${ }^{1,2}$ et \\ Idrissa Saidou MAHAMADOU ${ }^{1}$ \\ ${ }^{1}$ Département Sociologie et Economie Rurale, Faculté d'Agronomie de Université Abdou Moumouni de \\ Niamey, BP: 10960 Niamey, Niger. \\ ${ }^{2}$ Laboratoire d'Analyse et de Recherche en Soc iologie et Economie Rurales (LARSER), Faculté d'Agronomie \\ de l'Université Abdou Moumouni de Niamey, BP: 10960 Niamey, Niger. \\ *Auteur correspondant, E-mail : rizinat09@gmail.com, Tel : $0022791583247 / 0022799610566$. BP: \\ 10960 Niamey, Niger.
}

\begin{tabular}{lll}
\hline Received: 21-01-2021 & Accepted: 29-07-2021 & Published: 31-08-2021 \\
\hline RÉSUMÉ & &
\end{tabular}

Principale activité des ruraux nigériens, l'agriculture fait face à des problèmes récurrents de baisse de rendements conduisant à la paupérisation et à l'insécurité alimentaire des ménages qui l'exercent. Cette étude conduite à Karma vise la caractérisation des systèmes de production du mil et de niébé pluvial en vue de connaître leurs fonctionnements et leurs diverses interactions dans un contexte de changement climatique. Pour cela, une enquête a été conduite sur un échantillon de 90 ménages d'exploitants du mil et niébé pluvial. L'étude a montré que les systèmes de production sont regroupés en trois types selon la méthode d'Analyse en Composantes Principales couplée à la classification de Nuées dynamiques. Il s'agit du système de production agricole pure (Type A), du système agroforesterie (Type B) et du système agropastoral (Type C). Le type (A) occupe une superficie moyenne de 1,5 hectares. Il est scindé en système de cultures sèches simple et système de cultures sèches associé au maraîchage. Le type (B) s'effectue sur 2,18 hectares et le type (C) sur une superficie moyenne de 3,25 hectares. Ainsi, le type (C) est plus performant que le type (B) qui l'est plus que le type (A).

(C) 2021 International Formulae Group. All rights reserved.

Mots clés : Caractérisation, système de production, mil, niébé pluvial, Karma.

\section{Typology of farms producing millet and rain-fed cowpeas in the rural commune of Karma}

\begin{abstract}
Main activity of nigeriens' rural, agriculture faces recurring problems drop in yields leading to pauperization and food insecurity of the households that exert it. This study conducted in Karma aims to characterize millet and rain-fed cowpea production systems with a view to understanding their functioning and their various interactions to enable producers to better cope with climate change. For this, a survey was conducted on a sample of 90 households farms of millet and rain-fed cowpea. The study showed that production systems are grouped into three types according to the Principal Component Analysis method coupled with classification.
\end{abstract}


of dynamic clouds. These are the pure agricultural production system (Type A), the agroforestry system (Type B) and the agro-pastoral system (Type C). Type (A) occupies an average area of 1.5 hectares. It is split into a simple dry crop system and a dry crop system associated with market gardening. Type (B) takes place on 2.18 hectares and type $(C)$ on an average area of 3.25 hectares. Thus, type $(C)$ is more efficient than type (B) which is better than type (A).

(C) 2021 International Formulae Group. All rights reserved.

Keywords: Characterization, production system, millet, rain-fed cowpea, Karma.

\section{INTRODUCTION}

Le secteur agricole doit faire face au changement climatique tout en relevant deux autres défis. Il s'agit non seulement de doubler la production vivrière dans les pays en développement d'ici 2050 compte tenu de la croissance démographique et de l'évolution des régimes alimentaires mais aussi de jouer un rôle central dans l'action de réduction des gaz à effet de serre (FIDA, 2010). Le Groupe d'experts intergouvernemental sur l'évolution du climat (GIEC) met en garde contre des baisses de rendement des cultures allant de 10 à 25 pour cent, voire plus, qui pourraient se généraliser d'ici à 2050 (FAO, 2017). Ces prévisions sont plus perceptibles au Niger situé au cœur du Sahel. L'agriculture et l'élevage restent les principales activités économiques du pays avec une contribution d'environ $40 \%$ du produit intérieur brut et une occupation de $85 \%$ de la population active. Malgré son importance, le secteur agricole peine à se moderniser et reste largement tributaire des aléas climatiques (PAM, 2010).

De façon générale, le système de production agricole est la gestion dans le temps et dans l'espace des facteurs de production de manière à satisfaire le mieux possible les critères de l'unité de production tout en respectant les contraintes imposées en occurrence, les contraintes climatiques, économiques, sociales et foncières (JeanMarie, 2016). Selon Jouve (1992) c'est « un ensemble structuré de moyens de production (travail, terre, capital) combinés entre eux pour assurer une production végétale et/ou animale en vue de satisfaire les objectifs et les besoins de l'exploitant». En d'autres termes, s'intéresser au système de production revient à s'intéresser au fonctionnement et à la dynamique du sous-système de culture, du sous-système d'élevage et à la combinaison des deux sous-systèmes dans les exploitations (Cochet et Sophie, 2005).

En Afrique de l'Ouest, le Niger est cité parmi les principaux producteurs du niébé selon Yoka et al. (2014) et du mil (FAO, 2009). L'agriculture nigérienne est dominée par les cultures du mil et du niébé. Principale alimentation de la population rurale, le mil est une plante dont les exigences répondent aux pays du sahel comme le Niger. C'est pourquoi cette plante est cultivée dans toutes les régions agricoles du pays. Son apport en protéine dans l'alimentation des nombreux ménages ruraux fait du mil la céréale la plus cultivée et la plus consommée au Niger.

Les statistiques sur la production du mil au Niger de 1961 à 2016 montrent une forte augmentation aussi bien des quantités produites que des superficies emblavées. Ainsi, on passe de 776000 tonnes pour 1640100 ha en 1961 à 3886079 tonnes pour 7230228 ha en 2016 d'après Boubacar et al. (2019). Cependant sa production reste insuffisante pour satisfaire les besoins des populations à cause de faible rendement en milieu paysan, 500 à $600 \mathrm{Kg} / \mathrm{ha}$ en moyenne (ICRISAT, 2002).

S'agissant du niébé (Vigna Unguiculata L. Walp), c'est une plante herbacée bien adaptée au sahel dont les caractéristiques répondent au contexte climatique, à la correction de la fertilité du sol. Son potentiel productif permet de pallier le problème alimentaire du pays. Son intérêt particulier réside dans une adaptation à la sécheresse, un haut potentiel de fixation de l'azote atmosphérique, une tolérance aux hautes 
températures etc. (FAO, 2009). La baisse de rendement agricole et la précarité des mécanismes traditionnels de gestion des risques fragilisent les systèmes de production et de survie, de sorte qu'elles amènent les populations à développer localement des stratégies d'adaptation de court terme qui sont souvent dommageables à l'environnement et à la durabilité économique de leurs exploitations (Arsène, 2014).

L'importance des cultures en présence et les baisses de rendements observés conduisant à la paupérisation et à l'insécurité alimentaire des ménages font de cette étude une nécessité. L'objectif était de contribuer à la connaissance des caractéristiques des systèmes de production du mil et du niébé pluvial à Karma au Niger. La compréhension des exploitations agricoles de cette zone et des différents liens qui prévalent au sein des typologies demeurent capitales en vue d'améliorer les techniques de production du mil et niébé pluvial.

\section{MATERIEL ET METHODES Matériel}

Le matériel qui a permis de mener cette étude est constitué de fiches d'enquête réservées aux chefs des exploitations. Ces fiches portent sur l'identification du chef, les caractéristiques de l'exploitation agricole et la caractérisation des systèmes de production du mil et niébé pluvial. Le logiciel SPSS dans sa version 20 et Excel 2016 ont été utilisés pour la saisie et le traitement des données collectées afin d'effectuer des analyses statistiques et de concevoir des tableaux.

\section{Méthodes}

\section{Approches méthodologiques}

Deux approches combinées ont servi à élaborer la méthodologie. Il s'agit de la recherche documentaire destinée à l'exploitation des données secondaires au niveau des centres de documentation puis de la mairie de Karma et des enquêtes menées sur le terrain pour la collecte des données primaires au niveau des exploitants du mil et niébé pluvial.
L'approche méthodologique de l'analyse des exploitations agricoles consiste à: - donner la caractérisation des producteurs en vue de dégager les grands traits sur les exploitants dans leur intégralité. Ce qui nécessite des analyses statistiques descriptives simples pour élaborer des tableaux croisés.

-établir la typologie et la caractérisation des exploitations avec l'analyse des composantes principales (ACP) couplée à la méthode de classification en Nuées dynamiques. La démarche basée sur la méthode d'étude des exploitations élaborée par Capillon (1993) qui privilégie l'étude du fonctionnement de l'exploitation comme un pôle permettant d'analyser la diversification des systèmes de production a été prise en compte.

- évaluer la force du travail estimée en actifs agricoles (AA) est obtenue grâce à la formule suivante : $\mathrm{AA}=($ nombre d'hommes $)+0,75^{*}$ (nombre de femmes) $+0,5^{*}$ (nombre d'enfants de moins de 15 ans).

- élaborer le compte d'exploitation des différents types d'exploitations agricoles pour lequel certains paramètres ont été calculés dont entre autre :

- le produit brut en valeur (PBV en F CFA) équivaut à la valeur de la production annuelle totale multipliée par le prix unitaire au moment de la récolte. $\mathrm{PBV}=$ quantité de la production finale $(\mathrm{Q})$ * Prix Unitaire (PU).

- La valeur ajoutée brute (VAB en F CFA) est déterminée par la formule $: \mathrm{VAB}=\mathrm{PBV}$ - $\mathrm{CV}$ où $\mathrm{CV}$ représente les charges variables.

- La valeur ajoutée nette (VAN) donnée par la formule suivante: VAN = VAB Amortissements. (CILF, 1999)

- La marge brute qui est donnée par le produit brut en valeur (PBV) diminué de la valeur des charges variables $(\mathrm{CV})$. Sa formule s'écrit $\mathrm{MB}=\mathrm{PBV}-\mathrm{VCV}$.

$\mathrm{VCV}$ : Valeur des charges variables.

- La marge nette (MN) est obtenue par le produit brut en valeur auquel on soustrait toutes les dépenses engagées dans le processus de production (charges totales: variables et fixes). Sa formule est $\mathrm{MN}=$ $\mathrm{PBV}-\mathrm{VCT}$ 
VCT : Valeurs des charges totales (variables + fixes) (Gnanda et al., 2015).

- Le coût de production $(\mathrm{CP})=$ Charges variables $(\mathrm{CV})+$ Charges fixes $(\mathrm{CF})$ (JeanMichel, 1981).

- dégager l'analyse de corrélation entre les facteurs déterminants de production des différents types d'exploitations agricoles afin de distinguer le seuil des facteurs qui influencent significativement le niveau de production.

\section{Population cible et échantillonnage}

Le choix des personnes cibles a été fait sur la base de leurs activités et de leur statut social (chef du ménage). Ainsi, la personne enquêtée doit être un chef de ménage pratiquant l'agriculture sous pluie sur laquelle porte l'enquête. Et ceci, sans distinction de sexe.

Le choix des villages a été fait non seulement en fonction du nombre total d'agriculteurs du mil et niébé pluvial au sein d'un village (population mère) mais aussi de l'accessibilité de la zone (au plan sécuritaire). Pour déterminer la taille de l'échantillon, la formule de Schwartz (1995) été utilisée. Elle s'écrit : $n=\frac{t^{2} * p(1-p)}{e^{2}}$ où :

- $n=$ taille de l'échantillon;

- $t=$ constante issue de la loi normale centrée réduite selon un certain seuil de confiance (en général 95\% et $t=1,96$ ) ;

- $p=$ pourcentage des gens qui présentent le caractère observé (80\%);

- $e=$ marge d'erreur d'échantillonnage (traditionnellement fixée à 5\%).

Pour trouver l'effectif des souséchantillons dans chaque village retenu, la méthode de proportionnalité a été utilisée. Le taux d'échantillonnage ou de sondage encore appelé coefficient $\mathrm{k}$ a été calculé tel que: $\mathrm{k}=\mathrm{n} / \mathrm{N}$ où $\mathrm{n}$ est la taille de l'échantillon et $\mathrm{N}$ est la taille de la population totale. Avec ce taux, on aboutit à 19 personnes à enquêter pour le village de Moulko béné, 14 pour le village de Moulko ganda, 18 pour le village de Kanta et 39 enquêtés pour le village de karma repartis entre le quartier de Tarbou béné ( 22 personnes à enquêter) et Tarbou ganda (17 personnes à enquêter).
Les producteurs étaient enquêtés individuellement en fonction de leur disponibilité à travers les questionnaires.

\section{RESULTATS}

\section{Caractéristiques générales des agriculteurs du mil et niébé pluvial}

La quasi-totalité des agriculteurs enquêtés au sein de la commune rurale de Karma sont des hommes (98,9\%). L'âge moyen de ces exploitants est de 54 ans avec un écart type de 14,05 . Toutefois, le plus jeune a 30 ans et le plus âgé des exploitants a 80 ans. Le nombre d'années d'expériences en agriculture est en moyenne de 27 ans. La taille moyenne et le nombre de personnes de l'exploitation moyen sont respectivement de $10 \pm 4,390$ personnes et $6 \pm 3,494$ personnes. Le niveau d'instruction des chefs de ménage révèle 44,9\% de non instruits contre 39,3\% des exploitants ayant fréquenté l'école coranique. L'école traditionnelle est représentée par une poignée d'exploitants avec un niveau primaire de l'ordre de $10,1 \%$ et un niveau secondaire de $5,6 \%$. Il est à noter que l'ensemble des chefs d'exploitations enquêtés ont pour activité principale l'agriculture. La superficie moyenne de l'échantillon est de 6,6 $\pm 5,38$ hectares par chef d'exploitation (Tableau 1).

\section{Typologies des exploitations agricoles du mil et niébé pluvial}

Les variables présentant les plus fortes corrélations ont servi à la réalisation de l'analyse en composantes principales (ACP). $\mathrm{Au}$ total, onze (11) composantes ont été retenues initialement. Il s'agit : de la Rotation entre cultures sèches et maraîchage (1), de l'apport en fumure minérale (2), de l'apport en fumure organique (3), de la superficie en hectare (4), du nombre d'années d'expérience en Agriculture du chef de l'exploitation (5), de son âge (6), du nombre d'actifs agricoles employés au sein de l'exploitation (7), du nombre de personnes de l'exploitation (8), de la main d'œuvre salariée (9), des capitaux investis (10) et de la présence d'arbres appétés par les animaux au sein de l'exploitation (11). Ainsi, comme nous montre le Tableau 2, trois 
facteurs permettant d'expliquer à eux seuls plus de $67 \%(67,79 \%)$ des variations totales expliquées ont été retenus après analyse de la variance.

La première composante $(29,45 \%$ de la variabilité) a été considérée comme axe des caractéristiques organisationnelles en raison de ces variables composantes que sont la rotation entre cultures sèches et maraîchage, l'apport de la fumure minérale et celui de la fumure organique. La deuxième composante $(21,53 \%$ de la variabilité) formée principalement de la superficie en hectare, du nombre d'années d'expérience en Agriculture, de l'âge du chef de l'exploitation, du nombre d'actifs agricoles et du nombre de personnes de l'exploitation a été considérée comme axe des caractéristiques sociodémographiques. $\mathrm{La}$ troisième composante $(16,80 \%$ de la variabilité) formée de la Main d'œuvre salariale, des capitaux investis et de la présence d'arbres appétés par les animaux est considérée comme axe des aspects techniques de production (Tableau 3).

\section{Classification des exploitations agricoles du mil et niébé pluvial}

Selon la classification de Nuées dynamiques, ces trois axes retenus ont servi à regrouper les producteurs en trois classes homogènes. Les variables les plus déterminantes pour la classification des exploitants sont : les facteurs de production, les caractéristiques du ménage, les éléments du système de culture et le statut de l'exploitation. C'est ainsi que trois types de systèmes de production ont été détectés au niveau de la commune rurale de Karma selon ladite classification. Il s'agit du système de production agricole pure représenté par le type (A) destiné à la production végétale pure avec ou sans apport de fumure. Il occupe une superficie de 1,5 hectares et se trouve être présent sur les pentes, les glacis, les bas-fonds et les bordures de bas-fonds. Il est divisé en deux (2) sous-systèmes: le système de production à cultures sèches et le système de production associant cultures sèches et cultures irriguées. Le premier sous-système est un système dans lequel le producteur effectue les cultures de mil et niébé sous pluies. Le deuxième sous-système associe les cultures sèches du mil et niébé aux cultures irriguées. A ce niveau, l'argent issu de la vente des produits maraîchers vient renforcer l'achat d'intrants (engrais, produits phytosanitaires) favorables au bon développement des cultures sèches. Dans cette zone, on remarque la présence du système agroforesterie qui cadre avec les exploitants du type (B). Il est marqué par la présence d'arbres appétés par les animaux au sein de l'exploitation dans laquelle le producteur installe les cultures sèches. Il a pour finalité la production végétale et sylvicole. Il s'effectue sur une surface moyenne de 2,18 hectares et est observable sur les plateaux, les affleurements rocheux et les replats sommitaux. Le troisième système nommé agropastoral porte sur la production végétale et pastorale. Il associe l'agriculture du mil et niébé pluvial à l'élevage. Les cultures mises en place bénéficient alors d'une fertilisation organique apportée par les animaux élevés au sein de l'exploitation. Ce dernier système est propre aux exploitants du type (C) ressortis par la classification des Nuées dynamiques. Il couvre une superficie moyenne de 3,25 hectares et se contemple dans les interfluves.

Selon les types d'exploitations, La main d'œuvre salariale (F CFA) et les capitaux (F CFA) suivent un ordre décroissant. Du type (A) au type (C), Ils passent respectivement de 61500 et 441500 à 11572 et 62214 . L'importance du prix de la main d'œuvre salariée et sa faiblesse sont fonction des capitaux investis. Ainsi, plus le capital est grand plus la main d'œuvre salariée l'est. Tout de même, la main d'œuvre familiale est restée constante en fonction des types (A), (B) et (C). Elle est représentée par trois (3) actifs agricoles partout. La plus petite superficie 1,5 hectares est observée au niveau du type (A) alors que le type (C) enregistre la plus grande superficie qui de 3,25 hectares.

Les chefs des exploitations du type (A) sont plus âgés, possèdent le plus grand nombre de personnes dans leurs exploitations et ont un nombre d'années d'expérience en agriculture supérieur à celui des deux autres types. De (A) à (C) en passant par (B), la taille du ménage est respectivement de 20, 9 et 13 personnes. En ce 
qui concerne l'instruction, on note une absence totale du niveau supérieur et de l'alphabétisation chez tous les exploitants. Plus de $80 \%$ des enquêtés sont représentés par l'écrasante majorité de non instruits et les producteurs ayant fréquenté de l'école coranique. Le niveau primaire et le secondaire sont présents avec un pourcentage de moins de $10 \%$ chacun chez les types (B) et (C) tandis que le type (A) n'a que le niveau primaire à $12,5 \%$.

Les éléments de culture que sont l'assolement et la rotation évoluent au sein des types suivant un ordre identique pour le type (A) à 62,5\% et est composé essentiellement des cultures sèches que sont le mil et le niébé puis des cultures irriguées (tomates, oignons, laitues, aubergines et patate douce). A l'inverse, les exploitants des types (B) et (C) font plus d'assolement que de rotation.

Quant aux modes d'accès à la terre, la majorité des terres exploitées ont été acquises par héritage à 52\% par les exploitants du type (B) et à plus de $60 \%$ par ceux des types (A) et (C). S'en suit le gardiennage à $25 \%, 43 \%$ et $37 \%$ respectivement par les types (A), (B) et (C). L'achat n'est présent qu'au niveau du type (A) à $13 \%$. Il est quasi absent (2\%) chez les exploitants du type (C). La location n'est présente que chez les exploitants du type (B) à $5 \%$. On note une absence totale de prêt (Tableau 4).

\section{Compte d'exploitation des différents types de système de production}

L'analyse de la structure du coût de production indique que ce coût s'avère plus important chez les exploitants du type (A) et plus faible chez les exploitants du type (C). Tout de même, les résultats de production générés sont assez conséquents pour couvrir toutes les charges au niveau de tous les trois types d'exploitation. Malgré son coût de production élevé, le type (A) enregistre la plus petite valeur ajoutée nette (Tableau 5).

Dans le souci de comparer les résultats issus des trois types d'exploitation sur la même base, nous avons ramené quelques résultats du
Tableau 5 à la même superficie : à l'échelle d'un hectare. L'analyse du Tableau 6 montre que le type (C) investit moins (47926,36 F CFA) pour gagner plus $(102282,99 \mathrm{~F} \mathrm{CFA})$. L'indice de profitabilité donné par le ratio entre la marge nette $(\mathrm{MN})$ et le coût de production (CP) est de 2,09 au niveau du type(C). En d'autres termes, tout exploitant du type (C) qui investit 1 F CFA gagne 2,09 F CFA. Ainsi, le type (C) s'avère être plus performant que le type (B) qui a un gain de 1,59 F CFA qui l'est plus que le type (A) qui gagne 0,72 F CFA après chaque investissement d'un (1) F CFA selon les résultats du tableau 6 ci-dessous.

\section{Facteurs déterminants de la production}

La production moyenne annuelle du mil et niébé obtenue pour la campagne 2019 des trois types d'exploitations est de $2818,89 \mathrm{~kg}$. Cependant la répartition par type d'exploitations révèle des niveaux de disparité pour les quantités moyennes obtenues (Tableau 7).

Ainsi, pour le type (A), la main d'œuvre, les capitaux, la dose d'engrais et les produits phytosanitaires sont les principaux facteurs déterminants de la production. Au niveau de ces facteurs, la corrélation est forte pour la main d'œuvre et les produits phytosanitaires et moins forte pour les capitaux et la dose d'engrais au seuil $1 \%$. De ce fait, lorsque le niveau de tous ces facteurs augmente, le niveau de production augmente au sein des exploitations du type (A). Un effet contraire est observé au niveau du type (C) au même seuil de $1 \%$ avec une corrélation négative. Quant au type (B), deux facteurs ont été déterminants pour la production, il s'agit de la main d'œuvre et des capitaux. Les corrélations obtenues au seuil de 5\% entre ces deux facteurs et la production sont positives. Tout de même, la superficie, la quantité de semences, la dose d'engrais ainsi que les produits phytosanitaires sont corrélés positivement à la production pour tous les autres types mais n'influencent pas significativement le niveau de production. 
Tableau 1 : Caractéristiques générales des agriculteurs enquêtés.

\begin{tabular}{|c|c|c|c|c|c|}
\hline \multicolumn{6}{|c|}{ Caractéristiques quantitatives } \\
\hline \multirow{2}{*}{\multicolumn{2}{|c|}{ Variables }} & \multicolumn{4}{|c|}{ Catégories socioéconomiques } \\
\hline & & $\min$ & $\max$ & moy & Ecart type \\
\hline \multicolumn{2}{|l|}{ Age du chef d'exploitation (année) } & 30 & 80 & 53,91 & 14,056 \\
\hline \multicolumn{2}{|c|}{ Nombre d'années d'expérience en agriculture } & 3 & 69 & 27,66 & 16,476 \\
\hline \multicolumn{2}{|l|}{ Taille du ménage } & 3 & 25 & 10,12 & 4,390 \\
\hline \multicolumn{2}{|c|}{ Nombre de personnes de l'exploitation } & 1 & 16 & 6,76 & 3,494 \\
\hline \multicolumn{2}{|l|}{ Superficie (hectare) } & 0,8 & 28,10 & 6,60 & 5,38 \\
\hline \multicolumn{6}{|c|}{ Caractéristiques qualitatives } \\
\hline \multirow{4}{*}{$\begin{array}{l}\text { Niveau d'instruction des chefs de } \\
\text { ménage }\end{array}$} & \multirow{2}{*}{\multicolumn{5}{|c|}{$\begin{array}{c}\text { Pourcentage (\%) } \\
44,9\end{array}$}} \\
\hline & & & & & \\
\hline & $\begin{array}{l}\text { Ecole } \\
\text { coranique }\end{array}$ & \multicolumn{4}{|c|}{39,3} \\
\hline & Primaire & \multicolumn{4}{|c|}{10,1} \\
\hline \multirow{3}{*}{ Sexe du chef } & Secondaire & \multicolumn{4}{|c|}{5,6} \\
\hline & Masculin & \multicolumn{4}{|c|}{98,9} \\
\hline & Féminin & \multicolumn{4}{|c|}{1,1} \\
\hline
\end{tabular}

Tableau 2 : Variance totale expliquée.

\begin{tabular}{lcccccc}
\hline Composante & \multicolumn{3}{c}{ Valeurs propres initiales } & \multicolumn{3}{c}{$\begin{array}{c}\text { Extraction Sommes des carrés des } \\
\text { facteurs retenus }\end{array}$} \\
\cline { 2 - 7 } & Total & $\begin{array}{c}\text { \% de la } \\
\text { variance }\end{array}$ & \% cumulés & Total & $\begin{array}{c}\text { \% de la } \\
\text { variance }\end{array}$ & \% cumulés \\
\hline 1 & 3,24 & 29,454 & 29,454 & 3,24 & 29,454 & 29,454 \\
2 & 2,369 & 21,537 & 50,991 & 2,369 & 21,537 & 50,991 \\
3 & 1,848 & 16,802 & 67,792 & 1,848 & 16,802 & 67,792 \\
4 & 1,107 & 10,059 & 77,852 & & & \\
5 & 0,96 & 8,727 & 86,579 & & & \\
6 & 0,616 & 5,603 & 92,181 & & & \\
7 & 0,443 & 4,024 & 96,205 & & & \\
8 & 0,27 & 2,458 & 98,663 & & & \\
9 & 0,147 & 1,337 & 100 & & & \\
10 & $-8,99 \mathrm{E}-$ & $-8,17 \mathrm{E}-16$ & 100 & & & \\
& 17 & & & & & \\
11 & $-2,23 \mathrm{E}-$ & $-2,03 \mathrm{E}-15$ & 100 & & & \\
& 16 & & & & & \\
\hline
\end{tabular}

Méthode d'extraction : Analyse en composantes principales. 
Tableau 3 : Matrice des composantes.

\begin{tabular}{|c|c|c|c|c|}
\hline \multirow{3}{*}{\begin{tabular}{l}
\multicolumn{1}{c}{ Axes } \\
Caractéristiques \\
organisationnelles
\end{tabular}} & \multirow[t]{2}{*}{ Variables } & \multicolumn{3}{|c|}{ Composantes } \\
\hline & & $\mathrm{I}$ & $\overline{\text { II }}$ & III \\
\hline & $\begin{array}{l}\text { Rotation entre cultures sèches et } \\
\text { maraîchage }\end{array}$ & 0,93 & 0,339 & $-0,091$ \\
\hline & Apport en fumure minérale & 0,93 & 0,339 & $-0,091$ \\
\hline \multirow{6}{*}{$\begin{array}{l}\text { Caractéristiques } \\
\text { sociodémographiques }\end{array}$} & Apport en fumure organique & 0,93 & 0,339 & $-0,091$ \\
\hline & Superficie en hectare & $-0,412$ & 0,366 & $-0,341$ \\
\hline & $\begin{array}{l}\text { Nombre d'années d'expérience en } \\
\text { Agriculture }\end{array}$ & $-0,241$ & 0,714 & 0,43 \\
\hline & Age du chef de l'exploitation & $-0,367$ & 0,673 & 0,387 \\
\hline & Nombre d'actifs agricoles & $-0,429$ & 0,6 & $-0,478$ \\
\hline & $\begin{array}{l}\text { Nombre de personnes de } \\
\text { l'exploitation }\end{array}$ & $-0,278$ & 0,574 & $-0,508$ \\
\hline \multirow{3}{*}{$\begin{array}{l}\text { Aspects techniques de } \\
\text { production }\end{array}$} & Main d'œuvre salariale & 0,004 & 0,391 & 0,66 \\
\hline & Capitaux investis & 0,084 & 0,112 & 0,574 \\
\hline & $\begin{array}{l}\text { Présence d'arbres appétés par les } \\
\text { animaux }\end{array}$ & $-0,107$ & $-0,271$ & 0,347 \\
\hline
\end{tabular}

Tableau 4 : Caractéristiques des types d'exploitation.

\begin{tabular}{llccc}
\hline Eléments caractéristiques & Type (A) & Type (B) & Type (C) \\
\hline $\begin{array}{l}\text { Facteurs de } \\
\text { production }\end{array}$ & Main d'œuvre salariale (F CFA) & 61500 & 23368 & 11572 \\
& Capitaux (F CFA) & 441500 & 177675 & 62214 \\
& Main d'œuvre familiale (en actifs & 3 & 3 & 3 \\
& agricoles) & & & \\
& Superficie (hectare) & 1,5 & 2,18 & 3,25 \\
\hline & Age du chef d'exploitation (ans) & 67 & 49 & 55 \\
& Nombre de personnes de & 8 & 6 & 7 \\
& l'exploitation & & & \\
& Taille du ménage (personnes) & 20 & 13 & 9 \\
& Nombre d'années d'expérience en & 55 & 23 & 29 \\
Caractérisques & agriculture du Chef (ans) & & & \\
du ménage & Analphabète (\%) & 50,0 & 42,9 & 45,6 \\
& Ecole coranique (\%) & 37,5 & 38,1 & 40,4 \\
& Alphabétisation (\%) & 0,0 & 0,0 & 0,0 \\
& Primaire (\%) & 12,5 & 9,5 & 8,8 \\
& Secondaire (\%) & 0,0 & 9,5 & 5,3 \\
& Supérieur (\%) & 0,0 & 0,0 & 0,0 \\
\hline Présence d'assolement (\%) & 62,50 & 42,90 & 45,60 \\
& Absence d'assolement (\%) & 37,50 & 57,10 & 54,40
\end{tabular}




\begin{tabular}{llccc}
$\begin{array}{l}\text { Eléments du } \\
\text { système de } \\
\text { culture }\end{array}$ & Présence Rotation (\%) & 62,50 & 28,60 & 8,80 \\
\hline \multirow{4}{*}{$\begin{array}{l}\text { Statut de } \\
\text { l'exploitation }\end{array}$} & Personnel-achat (\%) & 37,50 & 71,40 & 91,20 \\
& Personnel-héritage (\%) & 13 & 0 & 2 \\
& Location (\%) & 63 & 52 & 61 \\
& Gardiennage (\%) & 0 & 5 & 0 \\
& Prêt (\%) & 25 & 43 & 37 \\
\hline
\end{tabular}

Tableau 5 : Compte d'exploitation selon les trois types de système.

\begin{tabular}{lccc}
\hline & $\begin{array}{c}\text { Type A (Superficie } \\
\text { moyenne: 1,5ha) }\end{array}$ & $\begin{array}{c}\text { Type B (Superficie } \\
\text { moyenne: 2,18ha) }\end{array}$ & $\begin{array}{c}\text { Type C (Superficie } \\
\text { moyenne: 3,25ha) }\end{array}$ \\
\hline Charges variables & & & \\
Petits matériels & 102276,75 & 82213,38 & 45688,37 \\
Engrais & 44525 & 23452,38 & 9773,68 \\
Produits phytosanitaires & 3481,25 & 840,48 & 303,51 \\
Semences & 102187,5 & 124571,43 & 90421,05 \\
Total Charges variables & $\mathbf{2 5 2 4 7 0 , 5}$ & $\mathbf{2 3 1 0 7 7 , 6 7}$ & $\mathbf{1 4 6 1 8 6 , 6 1}$ \\
Charges fixes & & & \\
Main d'œuvre salariée & 56719 & 21071 & 6544 \\
Amortissements & 6964,29 & 3207,48 & 3030,08 \\
Total Charges fixes & $\mathbf{6 3 6 8 3 , 2 9}$ & $\mathbf{2 4 2 7 8 , 4 8}$ & $\mathbf{9 5 7 4 , 0 8}$ \\
Produit Brut & 544875,00 & 663620,00 & 481636,42 \\
VAB & 292404,50 & 432542,33 & 335449,81 \\
VAN & 285440,21 & 429334,85 & 332419,73 \\
Coût de Production & 316153,79 & 255356,15 & 155760,69 \\
Marge brute & 292404,50 & 432542,33 & 335449,81 \\
Marge nette & 228721,21 & 408263,85 & 325875,73 \\
\hline
\end{tabular}

Tableau 6 : Comparaison des résultats ramenés à l'hectare.

\begin{tabular}{lccr}
\hline & Type A & Type B & \multicolumn{1}{c}{ Type C } \\
\hline Total Charges variables & 168313,67 & 105998,93 & 44980,4954 \\
Total Charges fixes & 42455,527 & 11136,917 & 2945,87077 \\
Produit Brut & 363250 & 304412,84 & 148195,822 \\
VAB & & & \\
VAN & 194936,33 & 198413,91 & 103215,326 \\
Coût de Production (CP) & 190293,47 & 196942,59 & 102282,994 \\
Marge brute & 210769,19 & 117135,85 & 47926,3662 \\
Marge nette (MN) & 194936,33 & 198413,91 & 103215,33 \\
VAN/CP & 152480,81 & 187277 & 100269,46 \\
MN/CP & 0,90 & 1,68 & 2,13 \\
& $\mathbf{0 , 7 2}$ & $\mathbf{1 , 5 9}$ & $\mathbf{2 , 0 9}$ \\
\hline
\end{tabular}


Tableau 7 : Corrélations entre la production moyenne et les facteurs déterminants.

\begin{tabular}{ccccccc}
\hline Variables & Superficie & $\begin{array}{l}\text { Main } \\
\text { d'œuvre }\end{array}$ & Capitaux & $\begin{array}{c}\text { Quantité } \\
\text { semences }\end{array}$ & Engrais & $\begin{array}{l}\text { Produits } \\
\text { phytosanitaires }\end{array}$ \\
\cline { 2 - 7 } & Sig & Sig & Sig & Sig & Sig & Sig \\
\hline Type (A) & 0,009 & $0,773^{* *}$ & $0,489^{* *}$ & 0,009 & $0,287^{* *}$ & $0,584^{* *}$ \\
& & & & & & \\
Type (B) & 0,175 & $0,206^{*}$ & $0,222^{*}$ & 0,175 & 0,129 & 0,042 \\
Type (C) & $-0,165$ & $-0,662^{* *}$ & $-0,502^{* *}$ & $-0,165$ & $-0293^{* *}$ & $-0,397 * *$ \\
& & & & & & \\
\hline
\end{tabular}

*La corrélation est significative au niveau 0,05 (unilatéral).

** La corrélation est significative au niveau 0,01 (unilatéral).

\section{DISCUSSION}

Le foncier est un facteur important pour les chefs d'exploitation. Il possède un caractère d'acquisition patrilinéaire et représente une richesse incontournable dans la pratique de l'agriculture mais aussi de l'élevage. L'étude a indiqué que l'héritage est le mode d'accès prédominant chez tous les types d'exploitations à plus de $50 \%$. Cependant, la pression démographique engendre un morcellement des terres conduisant à une diminution des superficies par exploitant (Marthe et Marie, 2004).

Cette étude fait ressortir une typologie des exploitations agricoles cloisonnée en trois portant les noms des types (A), (B) et (C). Ces types d'exploitations ont été spécifiés dans les résultats afin de révéler à la fois la différence qui caractérise chaque type d'exploitation et les éléments de même nature qui composent chacun des types (Kobrich et al., 2003; Topp et Mitchell, 2003). Certains auteurs qui ont mené des études similaires dans d'autres contrées ont obtenu plus de trois types. Ce nombre plus élevé que celui obtenu dans cette étude, est lié à la nature des facteurs de production pris en compte. Il s'agit de Johan (2002) qui a obtenu au nord de la Côte d'Ivoire quatre types de systèmes de production. Ce même auteur précise que les exploitations situées dans les bas-fonds n'ont pas de production satisfaisante. Il en est de même pour les résultats de cette étude. Graveline et al. (2004) ont obtenu une typologie composée de sept exploitations agricoles au sein de la vallée du Rhin supérieur alors qu'au Niger à la périphérie de la forêt protégée de Baban Rafi, Soukaradji et al. (2017) avaient obtenu quatre types d'exploitations agricoles.

Pendant que le nombre d'années d'expérience en Agriculture varie de 55 ans à 23 ans selon les types d'exploitations identifiés dans cette étude, il est de 22 ans au Bénin et reste constant chez tous les exploitants quel que soit leur type d'exploitation comme suggéré par Sossou et al. (2013). Cette variation serait due au fait que l'agriculture est introduite dans les habitudes des exploitants dès leur bas-âge (entre 7 ans et 10 ans). Le phénomène d'exode explique le plus petit nombre d'années d'expérience dans le domaine agricole à Karma.

Dans la zone de Dikodougou la culture de l'igname krenglè s'effectue sur une superficie moyenne de 0,87 ha et génère une VAN de $284723 \mathrm{~F} \mathrm{CFA/ha} \mathrm{avec} \mathrm{un} \mathrm{total} \mathrm{de}$ consommations intermédiaires de $133367 \mathrm{~F}$ CFA/ha selon Johan (2002) alors que dans notre étude, le type A qui associe le marâichage et qui s'apparente à cette étude génère une VAN de 190293 F CFA/ha. Une différence qui peut s'expliquer par le fait que les deux cultures en présence n'ont pas les mêmes finalités. L'igname est faite pour être vendue alors que les cultures du type A sont destinées plus à la subsistance qu'à la commercialisation des ménages qui les pratiquent. L'engrais organique est procuré par achat chez les types (A) et (B) mais est propre pour les exploitations 
du type (C). Il est complété par conséquent par les engrais chimiques comme l'urée et le NPK qui paraissent coûteux mais nécessaire pour le bon développement des cultures au vue de l'insuffisance de la fumure organique permettant de couvrir l'ensemble des unités d'exploitation. Le compost a une apparition récente et la non maîtrise de sa confection fait qu'il est utilisé d'une façon très négligeable. Des résultats pareils ont été obtenus par Jeanne et Antonin (2009) dans le sud-est de Madagascar puis Bazongo et al. (2015) dans la zone ouest du Burkina $\mathrm{Fa}$ so concernant l'utilisation des engrais.

Bien que le Niger soit un pays d'élevage par excellence, cette partie située dans la vallée du fleuve se caractérise par un élevage relativement peu développé. Ainsi, l'élevage pratiqué est donc de petites tailles et extensifs dans la quasi-totalité des cas. Il valorise les surfaces dans lesquelles il s'y trouve et s'adapte aux contraintes intrinsèques de la zone. Ces résultats sont semblables à ceux obtenus dans l'une des typologies de Graveline et al. (2004) en l'occurrence son type B2 du pays de Bade qui s'apparente à notre type (C). Cette similitude réside dans le gain pour l'épandage des déjections animales dont bénéficient les terres en présence. Tout de même, l'assolement est marqué par la présence de maïs grain à $37 \%$ pour le type $\mathrm{B} 2 \mathrm{~d}$ 'Alsace alors qu'il est caractérisé par la présence du mil et du niébé pluvial à $45,6 \%$ avec une faible rotation de 8,8\% au niveau du type (C) de Karma.

Au Burkina, Zoumana (2006) dans son étude sur les systèmes de production axée sur l'élevage obtint des résultats similaires à ceux de l'étude en ce qui concerne le niveau d'instruction des producteurs enquêtés. Ainsi, il s'agit de $43 \%$ de non instruits dans son étude contre $45,6 \%$ dans la nôtre. La différence réside au niveau supérieur où il enregistre $5 \%$ des chefs d'exploitations alors que dans notre étude on note l'absence totale de ce niveau. Tout de même, ces exploitations associent dans leur intégralité l'élevage aux pratiques de production végétale sur une superficie de 0,45 ha pour le mil et 0,11 ha pour le niébé.
Dans une étude faite sur la caractérisation des systèmes de production agricole dans la région de Dosso, le projet ANADIA.2 (2018) obtient un système dominant qu'est l'agriculture sous pluie. Ces résultats se rapprochent de ceux obtenus dans la présente étude. La différence réside au niveau du système de production pastorale qui est fait de façon isolé car n'associant pas l'agriculture. Cependant, cette étude ne fait pas cas d'une association de production agricole et sylvicole malgré l'existence d'une potentialité. Ils sont donc amputés de la valeur ajoutée apportée par la foresterie (N'Diaye Samba et al.,2012; Saidou et al., 2012) et l'élevage (Kouassi et al., 2019) dans leur dimension de fertilisation du sol d'autant plus que les arbres présents dans les exploitations du type (B) de Karma sont principalement composés de Faidherbia albida (Gao), Hyphaene thebaica (palmier doum) et Balanites aegyptiaca.

\section{Conclusion}

La présente étude a permis de caractériser différents systèmes de production des exploitants agricoles de Karma suivant une démarche mettant en relief les différents modes de gestion des unités de production. Elle nous a aussi permis de voir que les exploitants agricoles de cette zone adaptent un système de production rudimentaire de type (A) à 29,45\% malgré les diverses contraintes particulières rencontrées par la culture de mil et niébé pluvial. De la typologie des exploitations agricoles de production, l'étude a montré que le type (C) qui associe l'élevage à l'agriculture est plus performant que le type (B) spécifique au système agroforestier qui est à son tour plus performant que le type (A) qui est un système de production agricole pure.

\section{CONFLITS D'INTERETS}

Les auteurs déclarent qu'ils n'ont aucun conflit d'intérêts.

\section{CONTRIBUTIONS DES AUTEURS}

YAR: Principal rédacteur; SB : Supervision des travaux et rédacteur associé ; ISM : Apport lors du traitement des données. 


\section{REMERCIEMENTS}

Toute l'équipe du Laboratoire d'Analyse et de Recherche en Sociologie et Economie Rurales (LARSER) de la Faculté d'Agronomie de l'Université Abdou Moumouni de Niamey.

\section{RÉFÉRENCES}

ANADIA 2.0. 2018. Caractérisation des systèmes de production agricole dans la région de Dosso. Rapport n. 4, 48 pages.

Arsène D. 2014. Evaluation de la vulnérabilité et des stratégies d'adaptation des agriculteurs face au changement climatique dans le canton de Miandoum au Tchad. Mémoire de master en changement climatique AGRHYMET ; 82 pages.

Bazongo $\mathrm{P}$, Traoré $\mathrm{K}$, Traoré O, Bilgo A, Yelemou B, Sanon KB, Hien V, Nacro BH. 2015. Caractérisation des systèmes de production de Jatropha dans les exploitations agricoles de la zone Ouest du Burkina Faso. Int. J. Biol. Chem. Sci., 9(5): 2432-2445. DOI: http://dx.doi.org/10.4314/ijbcs.v9i5.15

Boubacar K, Hayyo H, Issa K. 2019. Culture du mil [Pennisetum glaucum (L) R. Br] et ses contraintes à la production: une revue. Int. J. Biol. Chem. Sci., 13(1): 503524.

DOI : http://dx.doi.org/10.4314/ijbcs.v13i1.40

Capillon A. 1993. Typologie des exploitations agricoles, contribution à l'étude régionale des problèmes techniques. Thèse de doctorat, Tome 1. Paris : INAPG p. 48.

CILF. 1999. Dictionnaire d'Agriculture. Français-anglais-allemand, 1011 pages.

Cochet H, Sophie D. 2005. Comprendre l'agriculture en région agricole : Question de méthode sur l'analyse en terme de systèmes de production. Institut National Agronomique Paris Grignon (INAPG), UER Agriculture Comparée et Développement Agricole : Paris ; p.18.

FAO. 2009. Les céréales au Niger - accent sur le mil et le sorgho. https://www.ajol.info/index.php/ijbcs/art icle/download/186797/176071
FAO. 2017. Stratégie de la FAO relative au changement climatique.

FIDA. 2010. Changement climatique : Stratégies. p 10-36 pages.

Gnanda IB, Bougouma-Yameogo VCM, Wereme/N'diaye A, Ouedraogo $\mathrm{T}$, Kaboré A, Lodoun B, Sinon B. 2015. L'embouche bovine dans les élevages du plateau Centrale du Burkina Faso: Résultats économiques d'une démarche de validation d'un référentiel technicoéconomique sur la spéculation. Int. $J$. Biol. Chem. Sci., 9(6):2648-2662. DOI: http://dx.doi.org/10.4314/ijbcs.v9i6.11

Graveline N, Rinaudo J-D. (BRGM), Geberth F, Segger V. (LEL). 2004. Analyse des systèmes de production agricole de la vallée du Rhin Supérieur. Rapport BRGM/RP-53171-FR.

ICRISAT. 2002. Résultat du projet P6 :19972001. Gestion intégrée des ennemis du mil. Niamey. p.36.

Jean-Marie D. 2016. Changement climatique et agriculture durable au Burkina Faso: Stratégies de résilience basées sur les savoirs locaux. Rapport d'étude, p.36.

Jean-Michel C. 1981. Larousse agricole. Directeur de l'Ecole nationale supérieure des industries agricoles et alimentaires, 1207 pages.

Jeanne G, Antonin P. 2009. Caractérisation de la diversité des systèmes d'exploitation agricole du Sud-Est de Madagascar. Mémoire en vue de l'obtention du diplôme d'AGRONOMIE GENERALE d'AgroParisTech, p.143.

Johan S. 2002. Analyse technique et économique des systèmes de production agricole au nord de la Côte d'Ivoire. Thèse de doctorat, Katholieke Universiteit Leuven, p. 286.

Jouve P. 1992. Le diagnostic du milieu rural de la région à la parcelle : Approche systémique des modes d'exploitation agricole du milieu. Montpellier: CNEARC, p.40.

Kobrich C, Rehman T, Khan M. 2003. Typification of farming systems for constructing representative farm models: two illustration of the application of 
multivariate analyses in Chile and Pakistan. Agricultural Systems, 76 (1): 141-157.

DOI:

https://EconPapers.repec.org/Repec:eee: agisys:v:76:y:20030:i:1:p:141-157

Kouassi YF, Gbogouri GA, N'Guessan KA, Bilgo A, Pascal Angui KT, Ama TJ. 2019. Effets de fertilisants organique et organo-minéral à la base de déchets végétaux et animaux sur la croissance et le rendement du soja (Glycine max (L.) Merrill) en one de savane de Côte d'Ivoire. Agronomie Africaine, 31(1): 1 12(20 19). DOI : https://www.ajol.info/index.php/aga/artic le/view/186708

Marthe D, Marie M. 2004. Pression foncière et nouvelles normes d'accès à la terre : vers une détermination de l'agriculture au sud Niger? Dossier no. 128. International institue for Environment and Development, Programme Zones Arides. p. 18.

N'Diaye Samba SA, Elhadji F, Tala G, Hank M, Camire C. 2012. Cordyla pinnata améliore les propriétés du sol et la productivité des cultures. Int. J. Biol. Chem. Sci., 6(2): 714-725. DOI: http://dx.doi.org/10.4314/ijbcs.v6i2.15

PAM. 2010 : « Chocs et vulnérabilité au Niger: Analyse des données secondaires », Rapport global, p 13-77. http://www.wfp.org/food-securiy

Saidou A, Balogoun I, Koné BCP, Gnangle B, Aho N. 2012. Effet d'un système agroforestier à karité (Vitellaria paradoxa c.f/ gaertn) sur le sol et le potentiel de production du maïs (Zea maize) en zone Soudanienne du Bénin. Int. J. Biol. Chem. Sci., 6(5): 2066-2082. DOI: http://dx.doi.org/10.4314/ijbcs.v6i5.16
Schwartz D. 2015. Le jeu de la science et du hasard. La statistique et le vivant H. L. Dans Population 1995/1 (Vol. 50), page 228. https://www.cairn.info/revuepopulation-1995-1-page-228.htm

Sossou CH, Lebailly P, Hinnou CL. 2013. Essai de typologie des exploitations agricoles axée sur le financement de la production agricole au Bénin. p 22.

Soukaradji B, Abdou A, Lawali S, Aboubacar I, Mahamane A, Saadou M. 2017. Typologie des exploitations agricoles familiales : cas de la périphérie de la forêt protégée de Baban Rafi du Niger. Int. J. Biol. Chem. Sci., 11(3): 1096-1112. DOI: http://dx.doi.org/10.4314/ijbcs.v11i3.14

Topp CFE, Mitchell M. 2003. Forecasting the environmental and socioeconomic consequences of changes in the Common Agricultural Policy. Agricultural Systems., $\quad \mathbf{7 6}(1)$ : 227-252. DOI: https://doi.org/10.1016/S0308521X(01)00113-5

Yoka J, Loumeto JJ, Djego JG, Akouango P, Epron D. 2014. Evolution des teneurs en éléments minéraux des feuilles de niébé. J. Apl. Biosc., 79: 6799-6807. DOI: http:dx.doi.org/10.4314/jab.v79i11

Zoumana T. 2006. Analyse de la diversité des systems de production agricole vers l'activité de production laitière: cas de Bobo-Dioulasso (Burkina Faso). Mémoire de fin d'études en vue de l'obtention du diplôme d'Ingénieur du Développement Rural, Option : Sociologie et Economie Rurales, Université Polytechnique de BoboDioulasso, p. 44. 